\title{
The Philosophical Orientation of Evidence-Based Practice and its Relation to Nursing
}

\author{
Arwa Alsadoon* \\ Department of Medical-Surgical science-Nursing College, King Saud Bin Abdulaziz University, Saudi Arabia \\ *Corresponding author: Arwa Alsadoon, Medical-Surgical science-Nursing College, King Saud Bin Abdulaziz University, \\ Saudi Arabia
}

\begin{tabular}{|c|c|}
\hline ARTICLE INFO & ABSTRACT \\
\hline Received: 幽 June 24, 2019 & Citation: Arwa Alsadoon. The Philosophical Orientation of Evidence-Based Practice and \\
\hline Published: 慧 July 02, 2019 & \\
\hline
\end{tabular}

\section{Introduction}

In 1980, the healthcare system was somewhat outdated, and many members of the public had a negative image of healthcare providers because providers were using outdated procedures that were not cost effective. As a result, researchers began to evaluate and assess the effectiveness of healthcare treatments [1]. Researchers evaluated the effectiveness of various treatments by using randomized controlled trials (RCT) during which treatments were compared to placebos. These studies moved health sciences into a new era during which healthcare providers started to use the results of these studies to help them determine the most effective and cost-effective treatments for their patients. This movement helped to improve the healthcare system by lowering the authority of individual opinion and increasing the value of "data-based studies" [2]. In Canada, evidence-based practice (EBP) first began in the McMaster Medical School as a paradigm to describe the strategy of clinical learning [2]. Shortly afterward, it became the paradigm for both EBP medical education and medical practice.

EBP first began as evidence-based medicine (EBM), which is defined as "the conscientious, explicit, and judicious use of the current best evidence in making decisions about the care of individual patients" [3]. Currently, confusion exists between both terms EBP and EBM because they have been used interchangeably. In addition, the definition of EBP is not very clear, and there are many questions regarding the foundations of EBP, such as how to define the best source of evidence and the definition of clinical expertise. After reading more about EBP, it was understood that EBP has different meanings for different people [2].

The term EBP has been expanded to various sciences including nursing. The Registered Nurses' Association of Ontario (RNAO) states that EBP offers support to nurses to help them provide quality nursing care. Furthermore, EBP helps nurses provide equitable care even when resources are in short supply. Nurses have reconstructed the definition of EBP in various ways for use in nursing science and nursing practice with a common theme of evidence derived from research. However, these definitions are inconsistent in terms of interventions, clinical expertise, and sources of evidence other than research. Furthermore, nursing literature uses the phrase evidence-based nursing (EBN), which is consistent with EBP in its use of evidence, but EBN itself has been defined in different ways that have resulted in variations and confusion. For example, Kessenich and Guyatt describe EBN as a philosophy of learning whereas Stetler and colleagues use a definition that is abstracted from evidence-based medicine.

\section{Problem Statement and Rational}

As a nurse educator, I want to improve the quality of healthcare by preparing nursing students to apply the best practices with their clients using the EBP concept. However, I have found it difficult to educate students about EBP due to the existence of multiple definitions and a lack of clarity regarding the terms used to describe the concept, as well as the presence of arguments accusing EBP of being restrictive by depending on evidence derived from quantitative studies that ignore the patients' values and circumstances. In order to be able to educate nursing students regarding the use of EBP, I need to gain a better understanding of the philosophical underpinnings of the concept. Thus, the goal of this paper is to explore the philosophical orientation of EBP by discussing the ontology and epistemology of its inception and how it is related to nursing. 


\section{Ontology}

Ontology is a branch of metaphysics that attempts to understand the nature of existence and humankind, and it consists of a set of concepts and principles of a domain that explain the properties and the relationship between them [4]. Ontology helps to answer questions such as "What is reality?" and "What is the relationship between existences?" To better understand EBP, it is necessary to examine the basic structure and assumptions of the concept. A review of the literature reveals that the ontology of EBP is mechanistic, reductionist, and objective [5,1]. A mechanistic view assumes that reality is objective and exists independently of human intentions, denying the importance of the personal and interpersonal sense of the world that consists of relationships between existence and is characterized by continuous changing (e.g., it is not a fixed object) [6].

It is an empirical view that separates the relationship and interactions aspect of the world, and it supports the reductionist view, which deconstructs the complex body into parts to study it $[5,7]$. The reductionist approach reduces the human body into parts and explains the whole body via each separate part. This approach is rooted in medicine - in medicine, complex problems are broken down into a simpler form in order treat the problems. This approach is rooted in the medical belief that each disease has a singular target for treatment [8]. Evidence-based practice relies on experimental studies, such as controlled studies, which reduce clinical problems to parts and study each part individually to enable meta-analyses. Nursing is a combination of both subjective and objective views [4]. Nursing science is built on the holistic view, which was distinguished by Aristotle, who describes it as the whole fostering the part. The assumption of this view is that things cannot be explained by knowing the parts [7].

That is consistent with the nursing assumption of humans, which views humans as complex and irreducible, and it also follows the goal of nursing that is aimed to operationalize the knowledge of unitary human beings who are in mutual interaction with their environments for the public well-being. To ensure the holistic view is applied in nursing, nursing knowledge comes from both physiology science and psychosocial science, which makes it difficult to identify a unique identity for nursing. Moreover, nursing itself has many philosophical positions that result in variations in knowledge-building strategies as well as nursing being described by people with various opinions and beliefs [9]. In summary, nursing is a science that attempts to build its knowledge in a way that helps it to capture the multi-dimensional needs of human beings, which makes the adoption of EBP difficult due to the reductionist view of the concept that subdivides patients.

\section{Epistemology}

Epistemology is derived from ontology, and it is concerned with the creation and dissemination of knowledge in certain domains
[10]. It helps to understand the theory of knowledge, in regard to its methods and scope, as well as to distinguish beliefs from opinion [4]. Epistemology provides answers to questions such as "What is the nature of knowledge?" and "What is the source of knowledge?" Exploring the epistemology of EBP would help to understand the beliefs that justify the knowledge foundation of the concept and its relation to the epistemology of nursing. EBP was created by the science of medicine, which is a leading science in human health and plays a vital role in guiding the health sciences [6]. The science of medicine is influenced by positivist thinking and the empirical approach, which relies on experimental studies as its only source of knowledge and randomized control trials (RCT) as evidence.

Consequently, the source of knowledge in the concept of EBP is the evidence obtained from experimental studies, which negates the value of qualitative research and ignores the patients' experiences. Even though the patients' preferences have been added to the definition of EBP, it is still not fully understood how best to involve patients in the application of the concept of EBP. The Canadian Nurses' Association (CNA) defines evidence as information obtained from research and practice, which involves experimental, non-experimental, expert opinion, and historical or experiential evidence (CNA, 2008). The definition of evidence includes qualitative research that is placed in the category of least influence in the hierarchy of evidence in the empirical approach as well as the EBP concept. Nurses using the EBP concept focus on providing a high quality of care to achieve better outcomes, and they have no authority to change patient care procedures to fit individual patients' needs (CNA, 2017). As result, nurses follow the EBP guidelines and provide care without the ability to change or adjust the plan to fit the patients' values and preferences.

The literature review discusses the complexity of nursing because it deals with human beings who have multi-dimensional needs that cannot be identified using one approach [9]. Consequently, Parse [11] suggests two paradigms, the totality and simultaneity paradigms, to guide nursing and enable nurses to adopt the holistic view. The paradigms are different, and neither is superior, but the differences between each one support one another to provide sufficient capacity to encompass all the nursing activities [4]. Thus, the two paradigms have different approaches that allow nurses to use different concepts to meet patients' unique needs. It is important to examine the concept of EBP to assess whether it can fit with these paradigms. The totality paradigm contains the oldest and most dominant epistemology concept, which is contemporary empiricism (postpositivism) [12]. Societal norms are highly valued in this paradigm [13]. According to Parse [11], the meta-paradigm concept of totality defines humans as physicalpsychosocial-spiritual beings who manipulate the environment in order to maintain homeostasis.

Humans are able to adapt and cope to meet their needs as well as interact with their environment to achieve goals [12]. The 
health meta-paradigm concept is defined as a dynamic state and the result of bio- psychosocial, and spiritual process [14]. These definitions help to understand how these paradigms helped create nursing beliefs that justify nursing knowledge. Under the totality paradigm, nursing goals focus on managing and treating sickness as well as preventing illness using the predefined norms. This paradigm provides frameworks to guide nurses to care for patients, cure diseases, prevent illness, and help patients reach and maintain balance to attain health goals by promoting the adaptation process. The totality paradigm prompts nurses to help patients manage illnesses, and nurses assist patients in meeting their needs using the problem-solving process, which includes planning, implementation, and evaluation. The problem-solving process is a method that comes from empirical approach and characterized by the objective view.

This process divides the problem into its components and deals with each component independently, which is consistent with EBP epistemology. However, the totality paradigm acknowledges the relationships between different variables and patients' health. Nurses use the problem-solving process during the assessment phase, after which they move to the evaluation phase, which is an on-going phase that monitors the progress of the patient and the effectiveness of nursing interventions [14]. The primary decision maker in this paradigm is the nurse, and that allows the nurse to modify the plan to fulfill the patients' needs and meet their preferences. Thus, the totality paradigm accepts the concept of EBP but acknowledges the need to add the nursing perspective and patient involvement. The simultaneity paradigm varies from the totality paradigm because it does not compare the patient (person) to predefined norms. Under this paradigm, humans are considered as open beings that have multi-dimensional, mutual interactions with the universe and continuously change in unpredictable ways. The health meta-paradigm is defined by each person's perspective and experiences. The health definition depends on each person's values and beliefs.

The simultaneity paradigm relies on the patients' perspective and experiences to justify knowledge. Under this paradigm, nursing is about helping people reach a quality of life that is defined based on the patients' perspectives using theories that are predefined by nurses. As Daly [12] states, "Nurses uncover their own beliefs in light of the assumptions about the human-universe-health process in light of the nursing theories." Therefore, the decision-maker in this paradigm is the person. Knowledge under this paradigm is abstracted from different methods because the simultaneity paradigm supports different ways of knowing, including subjective knowledge and constructed knowledge. Subjective knowledge is defined as knowledge that is intuitive and derived from personal experience. Constructed knowledge is a process of knowing where the person is the creator of knowledge, and it involves subjective and objective knowledge. The simultaneity paradigm depends on people's experience, beliefs, and values, whereas EBP is an objective approach that focuses on diagnosing, management, and outcomes, which minimizes people's involvement in their own care. Thus, EBP is incongruent with the philosophical underpinnings of the simultaneity paradigm.

\section{Conclusion}

Nursing science is unique because of the diverse theories and frameworks that form the structure of nursing, and all of the mentioned theories and frameworks share the concept of the human-universe-health interrelationship. This review provides an examination of the underlying values and philosophic foundations of EBP to determine how this concept helps to develop the knowledge needed to support the nursing practice. The EBP concept originated from scientific experiments to help identify the best practices to offer quality of care as well as provide costeffective treatments. However, Kuhn [15] states that a single theory that may be applicable to many groups is not the same for all of them because subspecialties are differently educated and focus on different applications for their research findings. Therefore, the EBP concept must continue to be examined because if it is used to successfully improve the effectiveness of medicine and/or medical treatment, its success in one field might not apply to other disciplines. Thus, a careful examination is needed to understand the concept and successfully adopt it.

Currently, there is a movement toward changing the term EBP to evidence-informed decision-making (EIDM). This new term allows for the inclusion of all types of studies to be used in finding the best evidence for practice. The use of EIDM will also ensure that patients' values and circumstances are considered when finding best evidence [16]. Excluding that, in order to make sure that EIDM overcomes the gaps found in EBP, the structure and philosophical orientation of EBP must be fully understood $[17,18]$.

\section{References}

1. Yates C (2013) Evidence-based practice: The components, history, and process. Counseling Outcome Research and Evaluation 4(1): 41-54.

2. Jennings B, Loan L (2001) Misconceptions among nurses about evidence-based practice. Journal of Nursing Scholarship 33(2): 121-127.

3. Sackett D (1996) Evidence-based medicine: What it is and what it isn't. British Medical Journal 312(7023): 71-72.

4. Barrett E (2002) What is nursing science?. Nursing Science Quarterly 15(1): 51-60.

5. Tosam M (2014) The role of philosophy in modern medicine. Open Journal of Philosophy 4(1): 75-84.

6. Holmes D, Murray S, Perron A, Rail G (2006) Deconstructing the evidence-based discourse in health sciences: Truth, power, and fascism. International Journal of Evidence-Based Healthcare 4(3): 180-186.

7. Beresford M (2010) Medical reductionism: Lessons from the great philosophers. QJM: An International Journal of Medicine 103(9): 721724.

8. Ahn A, Tewari M, Poon C, Phillips R (2006) The limits of reductionism in medicine: Could systems biology offer an alternative?. PLoS Medicine 3(6): e208.

9. Nyatanga L (2005) Nursing and the philosophy of science. Nurse Education Today 25(8): 670-674. 
10. Epistemology (2004) In Stanford Encyclopedia of Philosophy online.

11. Parse R (1987) Nursing science: Major paradigm, theories, and critiques. Philadelphia, PA: Saunders

12. Daly J (2005) Professional nursing: Concepts, issues, and challenges. New York.

13. Cody W (1995) About all those paradigms: Many in the universe, two in nursing. Nursing Science Quarterly 8(4): 144-147.

14. Mitchell G, Cody W (1992) Nursing knowledge and human science: Ontological and epistemological considerations. Nursing Science Quarterly 5(2): 54-61.

\section{ISSN: 2574-1241}

DOI: 10.26717/BJSTR.2019.19.003287

Arwa Alsadoon. Biomed J Sci \& Tech Res

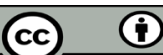

This work is licensed under Creative

Commons Attribution 4.0 License

Submission Link: https://biomedres.us/submit-manuscript.php
15. Kuhn T (1996) The structure of scientific revolutions (3rd ed.) Chicago, IL: University of Chicago Press pp. 43-51.

16. Woodbury M, Kuhnke J (2014) Evidence-based practice vs. evidenceinformed practice: What's the difference?. Wound Care Canada 12: 1821.

17. Canadian Nurses Association (1998) Positions statement: Evidenceinformed decision-making and nursing practice.

18. Mitchell G, Pilkington B (1990) Theoretical approaches in nursing practice: A comparison of Roy and Parse. Nursing Science Quarterly 3(2): 81-87.

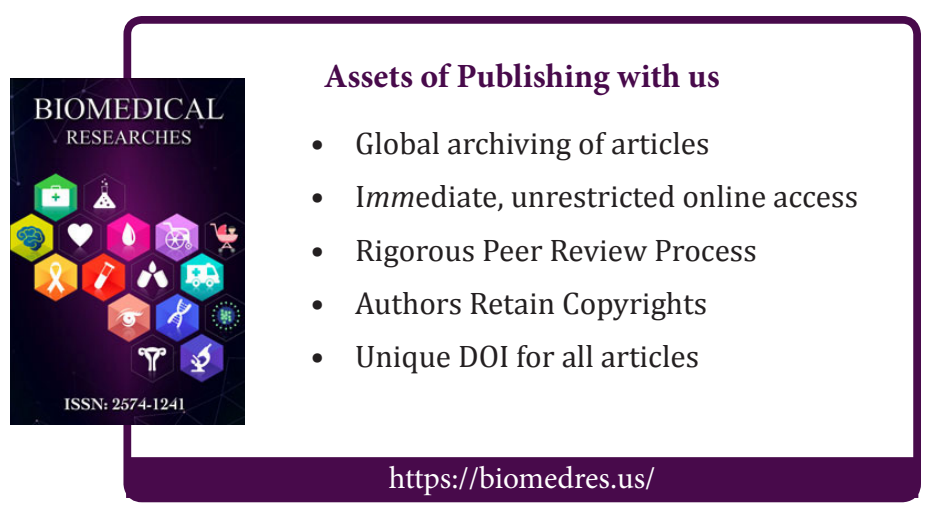

